\title{
Magnetoelastic Villari Effect in Ferrite Materials for Force and Stress Sensors Working in Low Magnetizing Field Region
}

\author{
M. Kachniarz ${ }^{a, *}$, A. Bieńkowski ${ }^{b}$, J. Salach $^{b}$ And R. SzewCZyK ${ }^{b}$ \\ ${ }^{a}$ Industrial Research Institute for Automation and Measurements, Al. Jerozolimskie 202, 02-486 Warsaw, Poland \\ ${ }^{b}$ Institute of Metrology and Biomedical Engineering, Warsaw University of Technology,
} św. A. Boboli 8, 02-525 Warsaw, Poland

\begin{abstract}
The following paper presents the original results of investigation on the magnetoelastic properties of ferrite materials magnetized in low field region, which could be utilized in development of force and stress sensors. The objects of investigation were two ferrite materials (manganese-zinc $\mathrm{Mn}-\mathrm{Zn}$ and nickel-zinc Ni-Zn). The magnetoelastic characteristics of the materials were investigated with the special measurement system, allowing measurement of magnetic parameters of the ferrite materials magnetized with low fields under the influence of the compressive stress. The obtained results indicate that there is a strong correlation between the magnetic properties of the material in low magnetizing field region, and applied mechanical stress, which allows development of the magnetoelastic stress or force sensor with ferrite core working in low magnetizing field region.
\end{abstract}

DOI: 10.12693/APhysPolA.133.1056

PACS/topics: 75.30.-m, 75.60.-d, 75.50.Gg, 75.80.+q, 07.10.Lw

\section{Introduction}

Metal-oxide materials known as ferrites are one of the most important groups of ferromagnetic materials in modern technology and science. They are often utilized as ferromagnetic cores for many inductive components in electronics including transformers, filters, chokes, etc. Ferrites are ceramic materials chemically composed of iron oxide $\mathrm{Fe}_{2} \mathrm{O}_{3}$ and one or more metallic elements (manganese, nickel, zinc, etc.) [1]. They are classified as ferrimagnetics, so their atoms are organized within two crystal sublattices exhibiting opposing magnetic moments. These atomic moments are not equal and they do not compensate each other completely, so the material still exhibits spontaneous magnetization, unlike antiferromagnetic.

The magnetoelastic Villari effect is a physical phenomenon involving changes of the magnetic properties of the ferromagnetic (or ferrimagnetic) material under the influence of the external mechanical stress [2-4]. This creates the possibility of ferrite materials utilization in measurements of mechanical force or stress. However, previous researches in this field were performed with high magnetizing fields, in saturation region [5]. It is very interesting to study the behaviour of ferrite materials under the influence of mechanical stress in low magnetizing fields. From the point of view of sensorical application, reduction of the amplitude of magnetic field magnetizing the core could result in significant reduction of the energy consumption of the sensor.

The following paper presents the results of studies on the magnetoelastic properties of ferrite materials in the

*corresponding author; e-mail: mkachniarz@piap.pl region of low magnetizing fields, known as the Rayleigh region, where magnetic characteristics of the material could be approximated with second degree polynomial equation.

\section{Rayleigh law of magnetization}

The Rayleigh law of magnetization was developed by British physicist lord John Rayleigh in 1887 [6]. Rayleigh discovered that for low magnetizing fields, significantly lower than coercive field in saturation region, the dependence between magnetic flux density $B$ and magnetizing field $H$ during the initial magnetization could be approximated with the second degree polynomial equation know as the Rayleigh law of magnetization [6]:

$$
B(H)=\mu_{0} \mu_{i} H+\mu_{0} \alpha_{\mathrm{R}} H^{2},
$$

where two material coefficients: initial magnetic permeability $\mu_{i}$ and Rayleigh coefficient $\alpha_{\mathrm{R}}$ are introduced and $\mu_{0}$ is magnetic permeability of free space.

The initial relative magnetic permeability $\mu_{i}$ is a parameter characterizing the reversible elastic deflections of the magnetic domain walls [7] resulting in linear changes of magnetic flux density Bunder the influence of magnetizing field $H$. The Rayleigh coefficient $\alpha_{\mathrm{R}}$ describes the irreversible translations of the domain walls, which results in nonlinear changes of magnetic flux density $B$ and is the source of magnetic hysteresis phenomenon in the Rayleigh region.

The magnetic hysteresis loop in the Rayleigh region (so-called Rayleigh loop) exhibits characteristic lenticular shape, which could be approximated with two intersecting parabolic curves described by the system of equations

$$
B(H)=\mu_{0}\left[\left(\mu_{i}+\alpha_{\mathrm{R}} H_{m}\right) H \pm \frac{\alpha_{\mathrm{R}}}{2}\left(H_{m}^{2}-H^{2}\right)\right],
$$

where $H_{m}$ is the amplitude of magnetizing filed. Intersection points of the parabolic curves are vertices of the Rayleigh loop. 
On the basis of previous researches [8] it may be expected that material coefficients introduced in the Rayleigh law of magnetization will be affected by the mechanical stress applied to the certain volume of the ferrite material. Thus, changes of the coefficients values can be utilized in force or stress measurement.

\section{Object of investigation}

The objects of investigation were two metal oxide materials exhibiting ferrimagnetic properties. The first one was based on manganese and zinc $(\mathrm{Mn}-\mathrm{Zn})$ and the second one was based on nickel and zinc (Ni-Zn). The chemical composition and basic physical and magnetic properties of the materials are presented in Table I. Magnetic parameters of the materials were measured for high magnetizing fields in the saturation region.

\section{TABLE I}

Chemical compositions of the investigated ferrite materials $\left(\mathrm{A}-\mathrm{Mn}_{0.58} \mathrm{Zn}_{0.36} \mathrm{Fe}_{2.06} \mathrm{O}_{4}\right.$, $\left.\mathrm{B}-\mathrm{Ni}_{0.36} \mathrm{Zn}_{0.67} \mathrm{Fe}_{1.97} \mathrm{O}_{4}\right)$ and their magnetic parameters measured in the saturation region.

\begin{tabular}{c|c|c}
\hline \hline Material & $\mathrm{A}$ & $\mathrm{B}$ \\
\hline average density $\left[\mathrm{mg} / \mathrm{m}^{3}\right]$ & 4.74 & 5.05 \\
\hline $\begin{array}{c}\text { maximum magnetic } \\
\text { flux density }[\mathrm{T}]\end{array}$ & 0.320 & 0.260 \\
\hline coercive field $[\mathrm{A} / \mathrm{m}]$ & 35 & 95 \\
\hline magnetic remanence $[\mathrm{T}]$ & 0.110 & 0.087 \\
\hline $\begin{array}{c}\text { initial magnetic } \\
\text { permeability }\end{array}$ & 1500 & 250 \\
\hline magnetostriction $[\mu \mathrm{m} / \mathrm{m}]$ & -0.31 & -2.04
\end{tabular}

Both materials were formed into frame-shaped samples. Each sample had two symmetrical columns, on which magnetizing (10 turns) and sensing (40 turns) coils were wound. The shape of the sample allowed to obtain closed magnetic circuit within the material and nearly uniform distribution of applied mechanical stress in the columns of the sample.

\section{Measurement system}

During the performed experiment, special digitally controlled measurement system was used. The schematic block diagram of the system is presented in Fig. 1. The setup is composed of two subsystems: system for investigation of magnetic characteristics of ferromagnetic materials and system for mechanical stress generation.

As a source of mechanical stress, acting on the sample, the oil hydraulic press was utilized. The force generated by the press is measured with strain-gauge force sensor. The results are transmitted to the $\mathrm{PC}$, where value of stress acting on the sample is calculated.

The measurement system for investigation of magnetic characteristics of ferromagnetic materials is described in detail in [9]. It is composed of PC with data acquisition

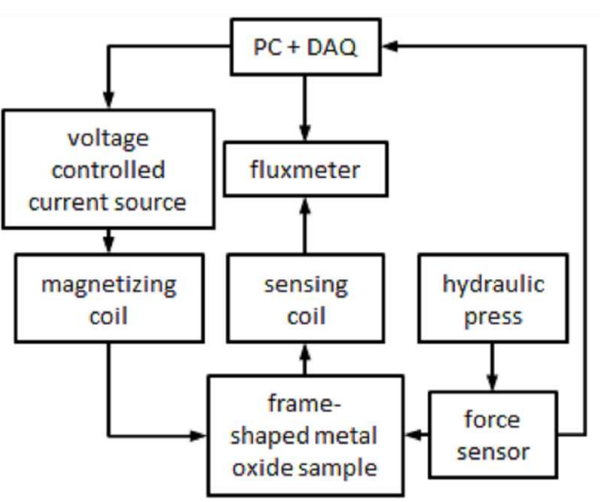

Fig. 1. Schematic block diagram of the measurement system.

card (DAQ) installed. The sinusoidal magnetizing waveform is generated by DAQ as voltage signal and then converted into current waveform with bipolar voltage controlled current source. The current waveform is driving the magnetizing coil, which results in generation of sinusoidal magnetizing field acting on the sample. As a result of magnetization process, the magnetic flux density within the material is changing, which leads to induction of voltage in the sensing coil. On the basis of obtained voltage waveform, the fluxmeter Lakeshore 480 is measuring the magnetic flux density within the material. The results are transmitted to the $\mathrm{PC}$.

\section{Experimental results}

Each of the investigated samples was subjected to the compressive stress $\sigma_{c}$ of value from $0 \mathrm{MPa}$ to $100 \mathrm{MPa}$. For each value of applied stress, the initial magnetization curve in the Rayleigh region and family of several Rayleigh loops were measured. The selected Rayleigh loops of investigated materials for constant amplitude of magnetizing field $H_{m}$ and different values of applied compressive stress $\sigma_{c}$ are presented in Fig. 2 and Fig. 3.

As it can be observed, for both investigated materials the hysteresis loops exhibit lenticular shape characteristic for the Rayleigh region. For $\mathrm{Mn}_{0.58} \mathrm{Zn}_{0.36} \mathrm{Fe}_{2.06} \mathrm{O}_{4}$ ferrite constant decrease of the maximum magnetic flux density $B_{m}$ and the surface area of hysteresis loop with the increase of applied compressive stress $\sigma_{c}$ is clearly seen, especially for high values of stress. In case of $\mathrm{Ni}_{0.36} \mathrm{Zn}_{0.67} \mathrm{Fe}_{1.97} \mathrm{O}_{4}$ material, the initial increase of $B_{m}$ and surface area of the loop occurs up to about $20 \mathrm{MPa}$ of compressive stress $\sigma_{c}$. This value of $\sigma_{c}$ corresponds to the so-called Villari point [2], which is the maximum point on the $B_{m}\left(\sigma_{c}\right)$ characteristic and is connected with the change of sign of the magnetostriction coefficient under the influence of stress [10]. In Mn-Zn ferrite this phenomenon is not observable due to the very low initial value of magnetostriction coefficient being only $0.31 \mu \mathrm{m} / \mathrm{m}$ (Table I), so eventual increase of $B_{m}$ would occur for very low value of applied stress, which was not measured. The observation of Villari point is possible due 


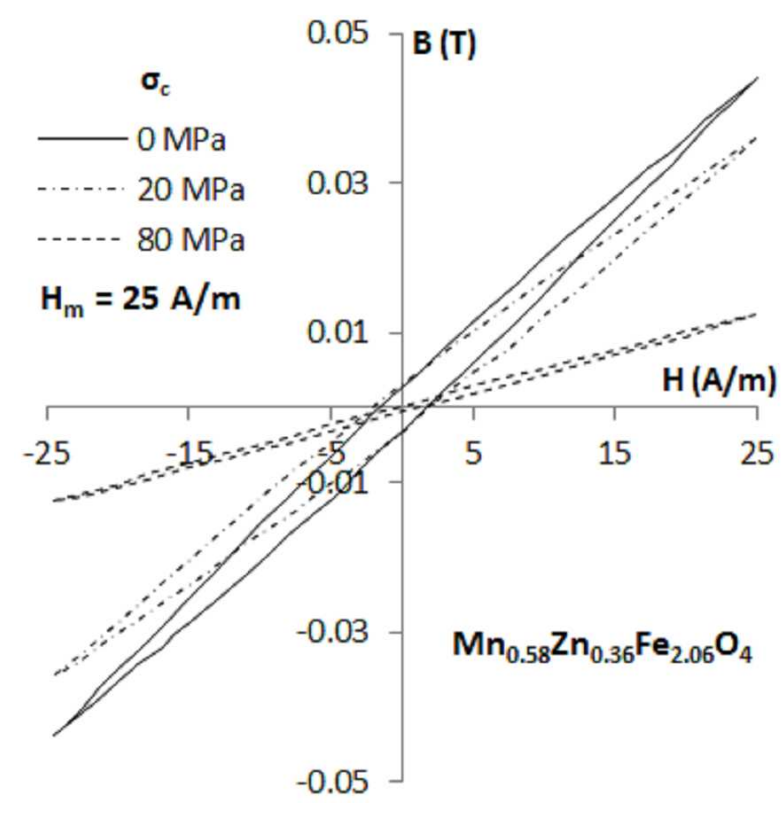

Fig. 2. Selected Rayleigh loops of $\mathrm{Mn}_{0.58} \mathrm{Zn}_{0.36} \mathrm{Fe}_{2.06} \mathrm{O}_{4}$ ferrite material for amplitude of magnetizing field $H_{m}=25 \mathrm{~A} / \mathrm{m}$.

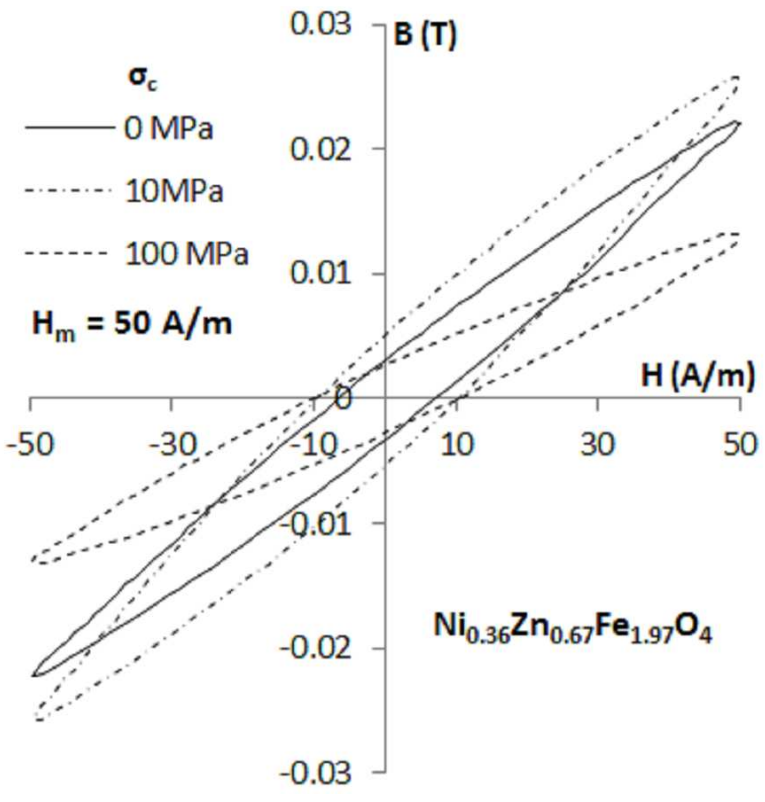

Fig. 3. Selected Rayleigh loops of $\mathrm{Ni}_{0.36} \mathrm{Zn}_{0.67} \mathrm{Fe}_{1.97} \mathrm{O}_{4}$ ferrite material for amplitude of magnetizing field $H_{m}=$ $50 \mathrm{~A} / \mathrm{m}$.

to the consistent direction of the vector of magnetizing field and the axis of applied mechanical stress $\sigma_{c}$.

On the basis of measured initial magnetization curves and the Rayleigh loops, the values of material coefficients: initial magnetic permeability $\mu_{i}$ and the Rayleigh coefficient $\alpha_{\mathrm{R}}$ were designated for each value of compressive stress $\sigma_{c}$ applied to the sample.

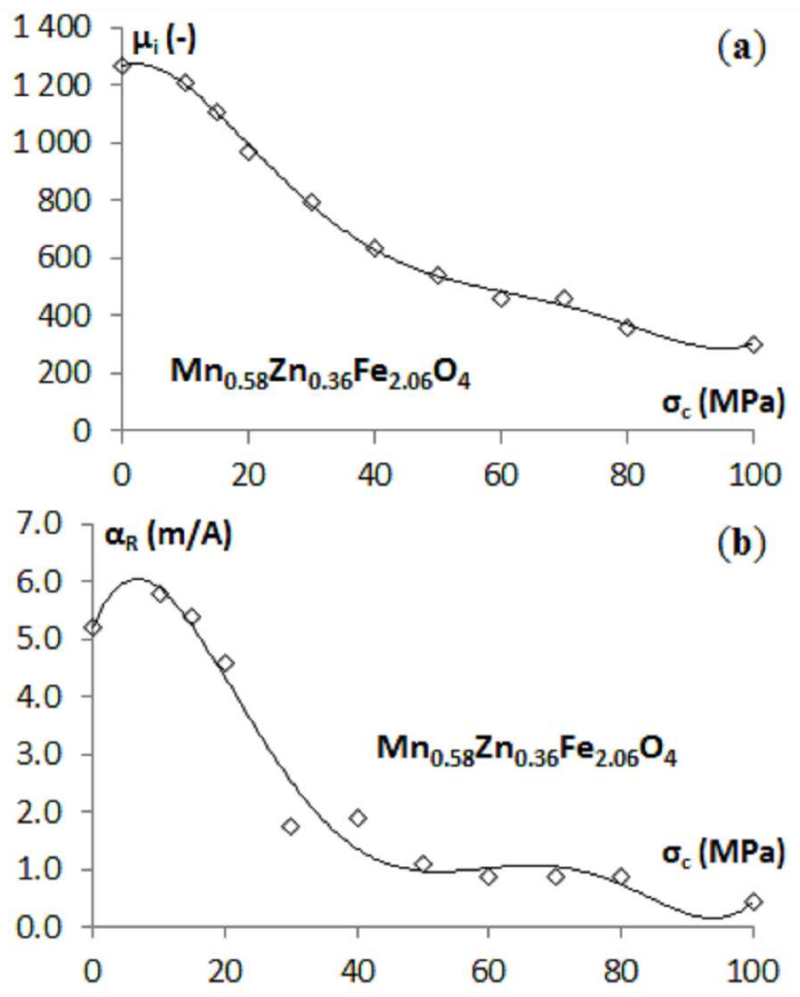

Fig. 4. Dependence of: (a) initial magnetic permeability $\mu_{i}$ and (b) Rayleigh coefficient $\alpha_{\mathrm{R}}$ on $\sigma c$ for $\mathrm{Mn}_{0.58} \mathrm{Zn}_{0.36} \mathrm{Fe}_{2.06} \mathrm{O}_{4}$ material (lines are polynomial approximations of the characteristics).

Figures 4 and 5 present the results of measurement of the material coefficients on the value of applied compressive stress $\sigma_{c}$ approximated with polynomial curves.

For both investigated materials, the behaviour of initial magnetic permeability $\mu_{i}$ under the influence of compressive stress $\sigma_{c}$ is similar. For high stress, there is a significant decrease of the value of $\mu_{i}$. The Villari point being maximum value of presented characteristic is observable only for $\mathrm{Ni}-\mathrm{Zn}$ material (Fig. 5a). As it was explained before, for $\mathrm{Mn}-\mathrm{Zn}$ material the Villari point is not seen due to the low value of magnetostriction coefficient. However both characteristics presented in Fig. 4a and Fig. 5a are generally similar.

On the other hand, significant differences are visible in characteristics of the Rayleigh coefficient $\alpha_{\mathrm{R}}$ dependence on the applied stress $\sigma_{c}$. The changes of $\alpha_{\mathrm{R}}$ in $\mathrm{Mn}-\mathrm{Zn}$ material are definitely stronger than in $\mathrm{Ni}-\mathrm{Zn}$ one. In both materials the maximum of $\alpha_{\mathrm{R}}\left(\sigma_{c}\right)$ characteristic is not located in the Villari point, but occurs for slightly higher stress. For $\mathrm{Ni}-\mathrm{Zn}$ ferrite there are two points about $30 \mathrm{MPa}$ and $40 \mathrm{MPa}$, which exhibit significantly lower values than neighbouring points. This situation is probably the result of measurement inaccuracy, as it is relatively hard to properly measure such low values of magnetic flux density as in the Rayleigh region. The mentioned points may also result from some kind of unidentified changes in the materials structure. 


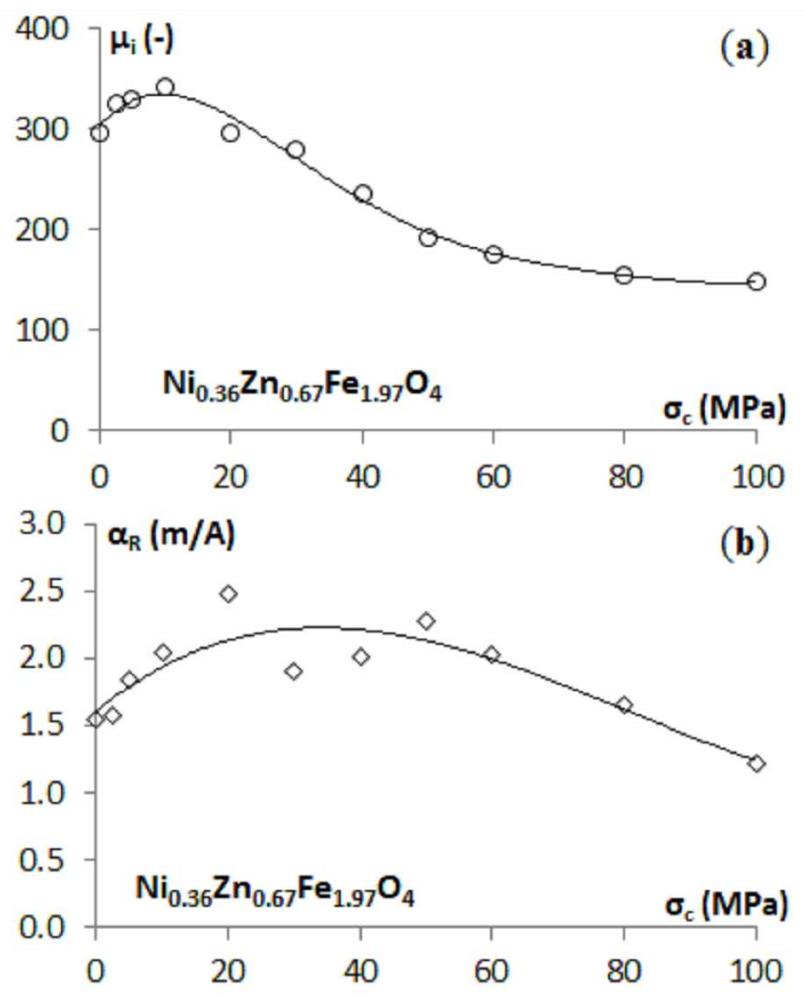

Fig. 5. Dependence of: (a) initial magnetic permeability $\mu_{i}$ and (b) Rayleigh coefficient $\alpha_{\mathrm{R}}$ on $\sigma_{c}$ for $\mathrm{Ni}_{0.36} \mathrm{Zn}_{0.67} \mathrm{Fe}_{1.97} \mathrm{O}_{4}$ material (lines are polynomial approximations of the characteristics).

\section{Conclusions}

In the paper the original results of investigation on magnetoelastic properties of $\mathrm{Mn}-\mathrm{Zn}$ and $\mathrm{Ni}-\mathrm{Zn}$ ferrite materials are presented. The measurement data indicate that there is a strong correlation between magnetic parameters of the ferrite material in low magnetizing fields and mechanical stress applied to it.

Comparing the two investigated materials it may be observed that their behaviour under the influence of compressive stress is generally similar. However there are several differences in the presented characteristics of the materials, especially in the dependence of Rayleigh coefficient on the applied stress. This may be the result of the imperfection of measurement methodology and further research should be carried out in order to validate the obtained results.

The results obtained during the investigation indicate that there is a possibility to utilize the ferrite materials for magnetoelastic cores of force and stress sensors working in low magnetizing field region.

\section{Acknowledgments}

This work was partially supported by the statutory funds of Institute of Metrology and Biomedical Engineering, Warsaw University of Technology (Poland).

\section{References}

[1] C.B. Carter, M.G. Norton, Ceramic Materials: Science and Engineering, Springer, New York 2013.

[2] A. Bieńkowski, J. Magn. Magn. Mater. 215-216, 231 (2000).

[3] D. Jackiewicz, J. Salach, R. Szewczyk, A. Bieńkowski, Acta Phys. Pol. A 126, 392 (2014).

[4] D.C. Jiles, J. Phys. D Appl. Phys. 28, 1537 (1995).

[5] S.H. Song, C.C.H. Lo, S.J. Lee, S.T. Aldini, J.E. Snyder, D.C. Jiles, J. Appl. Phys. 101, 09C517 (2007).

[6] Lord Rayleigh, Philos. Mag. 23, 225 (1887).

[7] B. Bertotti, Hysteresis in Magnetism for Physicists, Material Scientists and Engineers, Academic Press, San Diego 1998.

[8] R.R. Birss, IEEE Trans. Magn. 7, 113 (1971).

[9] T. Charubin, M. Urbański, M. Nowicki, in: Advances in Intelligent Systems and Computing, Vol. 543, Eds. R. Szewczyk, M. Kaliczyńska, Springer, Berlin 2017, p. 593.

[10] P. Svec Sr., R. Szewczyk, J. Salach, D. Jackiewicz, P. Svec, A. Bieńkowski, J. Hosko, J. Electr. Eng. 65, 259 (2014). 